\title{
Symmetric Einstein Spaces and Spectral Geometry
}

\section{HAROLD DONNELLY}

\author{
Communicated by S. S. CHERN
}

Introduction. Let $M^{n}$ be an $n$-dimensional Riemannian manifold and $\triangle$ its Laplacian. If $F_{p}(x, y, t)$ is the fundamental solution to the associated heat equation, then we have the asymptotic expansion

$$
\operatorname{Tr} F_{\nu}(x, x, t)=(4 \pi t)^{-n / 2}\left(\left(\begin{array}{l}
d \\
p
\end{array}\right)+a_{1, p} t+a_{2, p} t^{2}+\cdots\right) .
$$

The integrals $\int a_{i, p}$ are spectral invariants in the sense that if $M$ and $N$ have the same spectrum on $p$-forms, then $\int_{M} a_{i, p}=\int_{N} a_{i, p}$. Patodi [4] calculated $a_{1, p}$ and $a_{2, p}$ and gave some interesting geometric applications.

In this paper we prove using the coefficient $a_{3, p}$ that if an Einstein manifold $M$ has the same spectrum as a symmetric space, then $M$ is a symmetric space.

Invariants on Einstein manifolds. It is well known that the coefficients $a_{3, p}$ are invariant polynomials in the components of the curvature tensor and its covariant derivatives. Further, the $a_{3, p}$ are of order six in the derivatives of the metric. A basis for these invariants may be computed using $\mathrm{H}$. Weyl's invariance theorem [1]. His theorem says that the invariant polynomials are contractions in the components of the curvature tensor and its covariant derivatives. Taking into account the curvature identities and Bianchi identities, we obtain seventeen independent invariants on a generic Riemannian manifold. They are: $\tau|R|^{2}, \tau|\rho|^{2}, \tau^{3}$,

$$
\begin{aligned}
& \sum R_{i j i k} R_{i l m l} R_{k v m v}, \quad \sum R_{i j i k} R_{l v m v} R_{i l k m}, \\
& \sum R_{i j i k} R_{i l m v} R_{k l m v}, \quad \sum R_{i j k i} R_{i j m v} R_{k l m v}, \\
& \sum R_{i j k l} R_{i m k v} R_{j m l v}, \quad|\nabla \tau|^{2}, \quad|\nabla \rho|^{2}, \quad \Delta \Delta \tau \\
& |\nabla R|^{2}, \quad \sum R_{i j i k, l} R_{m j m l, k}, \\
& \tau \Delta \tau, \quad \sum R_{i j i k} R_{j l k l, m m}, \quad \sum R_{i j i k} R_{i l k m, l m}, \quad \sum R_{i j k l} R_{i j k l, m m}
\end{aligned}
$$


Here $\tau$ denotes the scalar curvature, $\rho$ denotes the Ricci tensor, $\nabla$ stands for covariant differentiation, $\triangle$ is the Laplacian, and the "," denotes covariant differentiation. After reduction by the Einstein condition, we have seven linearly independent invariants $\tau^{3}, \tau|R|^{2},|\nabla R|^{2}, \triangle\left(|R|^{2}\right), X=\sum R_{i j k l} R_{i j m v} R_{k l m v}$, $Y=\sum R_{i j k_{l} l} R_{i m k v} R_{i m l_{v}}, \Delta \triangle \tau$.

Integral relations on Riemannian manifolds. Some useful integral relations among our invariants were given in [5].

First, using Green's theorem, one obtains equation (2.14) of [5] which reduces in the case on an Einstein manifold of dimension $n$ to:

$$
\int Y=\int\left[\frac{1}{4}|\nabla R|^{2}-\frac{1}{4} X+\frac{1}{2} \frac{\tau}{n}|R|^{2}\right]
$$

Also for $n \leqq 6$ we have the following:

$$
\begin{aligned}
\chi(M) & =\frac{1}{384 \pi^{3}} \int\left[\frac{1}{9} \tau^{3}-\tau|R|^{2}-8 Y+4 X\right] \quad n=6 \\
0 & =\frac{1}{384 \pi^{3}} \int\left[\left(1-\frac{12}{n}+\frac{40}{n^{2}}\right) \tau^{3}+\left(3-\frac{24}{n}\right) \tau|R|^{2}-8 Y+4 X\right] \\
& n<6 .
\end{aligned}
$$

The first equation follows directly from (5.15) of [5] using the Einstein condition. The second follows similarly using the fact that $\chi(M \times T)=0$, where $T$ is an appropriate flat torus.

The coefficient $a_{3, p}$. By difficult calculations Sakai [5] derived a formula for $a_{3,0}$ which in the Einstein case reduces to:

(3)

$$
\begin{aligned}
a_{3,0}= & \frac{1}{6 !}\left[\tau^{3}\left(\frac{5}{9}-\frac{2}{3 n}-\frac{16}{63 n^{2}}\right)\right. \\
& +\tau|R|^{2}\left(\frac{2}{3}+\frac{136}{105}\left(\frac{1}{n}\right)\right) \\
& \left.+\frac{3}{5}|\nabla R|^{2}+Y\left(\frac{-896}{315}\right)+X\left(\frac{-104}{315}\right)\right] \\
& +\operatorname{div} V
\end{aligned}
$$

where $V$ is some vector field.

Theorem. Let $M^{n}$ be an Einstein manifold which has the same spectrum on p-forms as a symmetric space $N$. Then $M$ is symmetric.

Proof. Patodi [4] has shown that $N$ is Einstein and $\int_{M} \tau^{3}=\int_{N} \tau^{3}, \int_{M} \tau|R|^{2}=$ $\int_{N} \tau|R|^{2}$ since $\tau$ is a constant. 
Now first suppose $n \leqq 5$. Then from (1), (2), (3) we have

$$
\begin{gathered}
\int_{M} Y+\frac{1}{4} X-\frac{1}{4}|\nabla R|^{2}=\int_{N} Y+\frac{1}{4} X-\frac{1}{4}|\nabla R|^{2} \\
\int_{M}-2 Y+X=\int_{N}-2 Y+X \\
\int_{M}-\left(\frac{896}{315}\right) Y-\left(\frac{104}{315}\right) X+\frac{3}{5}|\nabla R|^{2}=\int_{N}-\left(\frac{896}{315}\right) Y-\left(\frac{104}{315}\right) X+\frac{3}{5}|\nabla R|^{2} .
\end{gathered}
$$

Algebra yields $\int_{M}|\nabla R|^{2}=\int_{N}|\nabla R|^{2}=0$, so $M$ is symmetric.

Now let $n \geqq 6$. Set

$$
a_{3, p}=C_{1}(d, p)|\nabla R|^{2}+C_{2}(d, p) X+C_{3}(d, p) Y+L\left(\Delta|R|^{2}, \tau^{3}, \tau|R|^{2}, \Delta \Delta \tau\right)
$$

Let $R_{m}$ denote the restriction map $V_{m+1}{ }^{6} \rightarrow V_{m}{ }^{6}$ which restricts an invariant of order six to a manifold of one lower dimension. Peter Gilkey [2] has shown that for $m \geqq 6, R_{m}$ is an injection. By considering manifolds of the form $M^{d_{1}} \times$ $T^{d-d_{1}}$ we deduce:

$$
C_{i}(d, p)=\sum_{a+r=p}\left(\begin{array}{c}
d-d_{1} \\
q
\end{array}\right) C_{i}\left(d_{1}, r\right)
$$

for $d_{1} \geqq 6$.

Using equation (1) we get

$$
\begin{aligned}
\int_{M}\left[C_{1}(n, p)+\frac{1}{4} C_{3}(n, p)\right]|\nabla R|^{2}+\int_{M}\left[C_{2}(n, p)-\frac{1}{4} C_{3}(n, p)\right] X \\
\quad=\int_{N}\left[C_{1}(n, p)+\frac{1}{4} C_{3}(n, p)\right]|\nabla R|^{2}+\int_{N}\left[C_{2}(n, p)-\frac{1}{4} C_{3}(n, p) \mid \dot{X}\right.
\end{aligned}
$$

We claim that as $p$ varies this gives at least two linearly independent equations. By (4) it suffices to verify this on six-manifolds. But on six-manifolds the fantastic cancellation of [3] yields for the alternating sum of the equations (5):

$$
\int_{M}-|\nabla R|^{2}+3 \int_{M} X=\int_{N}-|\nabla R|^{2}+3 \int_{N} X
$$

The formula (3) for $p=0$ yields

$$
\int_{M}-7|\nabla R|^{2}+24 \int_{M} X=\int_{N}-7|\nabla R|^{2}+24 \int_{N} X
$$

This gives the result. 


\section{REFERENCES}

1. Marcel Berger, Le spectre d'une variete Riemannienne, Springer, New York, p. 76.

2. Peter Gilkey, Private conversation.

3. V. K. Patodi, Curvature and the eigenforms of the Laplace operator, J. Differential Geometry 5 (1971), 233-249.

4. - Curvature and the fundamental solution of the heat operator, J. Indian Math. Soc. 34 (1970), 269-285.

5. T. SAKAI, On eigenvalues of Laplacian and curvature of Riemannian manifold, Tohuku Math. J. 23 (1971), 589-603.

Massachusetts Institute of Technology

Date communicated: February 5, 1974 